\title{
TWISTED CONJUGACY IN FREE PRODUCTS
}

\author{
DACIBERG GONÇALVES, PARAMESWARAN SANKARAN, AND PETER WONG
}

\begin{abstract}
Let $\phi: G \rightarrow G$ be an automorphism of a group which is a free-product of finitely many groups each of which is freely indecomposable and two of the factors contain proper finite index characteristic subgroups. We show that $G$ has infinitely many $\phi$-twisted conjugacy classes. As an application, we show that if $G$ is the fundamental group of a three-manifold that is not irreducible, then $G$ has property $R_{\infty}$, that is, there are infinitely many $\phi$-twisted conjugacy classes in $G$ for every automorphism $\phi$ of $G$.
\end{abstract}

\section{INTRODUCTION}

Let $G$ be an infinite group. Given an automorphism $\phi: G \rightarrow G$, one has an action of $G$ on itself, known as the $\phi$-twisted conjugation, defined as $g . x=g x \phi\left(g^{-1}\right)$. The orbits of this action are the $\phi$-twisted conjugacy classes. Let $\mathcal{R}(\phi)$ denote the orbit space. We denote by $R(\phi)$ the cardinality of $\mathcal{R}(\phi)$ if it is finite, and, when $\mathcal{R}(\phi)$ is infinite we set $R(\phi):=\infty$ and $R(\phi)$ is called the Reidemeister number of $\phi$. One says that $G$ has the $R_{\infty}$-property, or that $G$ is an $R_{\infty}$-group, if $R(\phi)=\infty$ for every automorphism $\phi$ of $G$. The notion of Reidemeister number first arose in the Nielsen-Reidemeister fixed point theory. Classifying (finitely generated) groups according to whether or not they have the $R_{\infty}$-property is an interesting problem and has emerged as an active research area that has enriched our understanding of finitely generated groups.

The fundamental group of a closed connected three-dimensional manifold is an important invariant of the manifold as it carries a lot of information concerning its topology. The main motivation for this work is to understand which manifolds have the property that their fundamental groups have the $R_{\infty}$-property. We have not been able to completely answer this question. However, we obtain a very general result showing that a wide class of groups have the $R_{\infty}$-property. This yields a partial answer, to the above question covering a large class of compact three-manifolds.

Recall that a closed connected three dimensional manifold $M$ is said to be prime if $M=M_{1} \# M_{2}$ implies that at least one of the $M_{i}$ is a 3 -sphere. One says that $M$

Date: January 22, 2020.

2010 Mathematics Subject Classification. 20E45, 22E40, 20E36

Key words and phrases: Twisted conjugacy, three-manifolds, free product of groups.

The first author is partially supported by Projeto Temático-FAPESP Topologia Algébrica, Geométrica e Diferencial 2016/24707-4 (São Paulo-Brazil). The first and third authors thank the IMSc (August 2018) and the CMI, Chennai (December 2019), for their support during their visits. Second and third authors thank the IME-USP, São Paulo for its support during the authors' visit in February 2019. 
is irreducible if every embedded 2-sphere is the boundary of a 3-disk in $M .{ }^{1}$ Every irreducible manifold is prime, but the converse is not true: $\mathbb{S}^{2} \times \mathbb{S}^{1}$ is an example of a prime manifold which is not irreducible. If $M$ is irreducible and has infinite fundamental group, then the sphere theorem (due to C. D. Papakyriakopoulos) implies that $M$ is a $K(\pi, 1)$ space. A fundamental result in three-manifold theory is that every closed connected orientable 3-manifold $M$, can be expressed as a connected sum: $M \cong M_{1} \# \cdots \# M_{k}$ where each $M_{j}$ is prime (and not the 3 -sphere). Moreover the decomposition is unique (up to reordering of the factors). When $M$ is non-orientable, one still has a prime decomposition. However, the uniqueness part fails. If $P$ is the non-trivial $\mathbb{S}^{2}$-bundle over $\mathbb{S}^{1}$, then $P \# N=$ $\left(\mathbb{S}^{2} \times \mathbb{S}^{1}\right) \# N$ when $N$ is non-orientable. In view of this, in the case when $M$ is nonorientable, one may assume that none of its prime factors $M_{i}$ is $\mathbb{S}^{2} \times \mathbb{S}^{1}$. With this restriction the uniqueness part is valid. See [H1, Chapter 3]. As for any finitely generated group, $\pi_{1}(M)$ may be decomposed as a free product of groups $\pi_{1}(M)=G_{1} * \cdots * G_{r}$ where each $G_{i}$ is freely indecomposable. It turns out that $r=k$ and after a reordering of indices $G_{i}=\pi_{1}\left(M_{i}\right), 1 \leq i \leq k$.

Our main result is the following:

Theorem 5. Let $M$ be a non-prime compact connected three-manifold. Then $\pi_{1}(M)$ has the $R_{\infty}$-property.

The algebraic result which leads to the above as a consequence is the following.

Theorem 1. Let $k \geq 2$. Suppose that $G=G_{1} * \cdots * G_{k}$ where (i) each $G_{i}$ is freely indecomposable, and, (ii) $G_{i}$ has a proper characteristic subgroups of finite index for $i=$ 1,2. Then $G$ has the $R_{\infty}$-property.

The main tool used in the proof of Theorem 1 is Kuroš subgroup theorem. It is wellknown that no group is both a nontrivial free product and a nontrivial direct product. See [LS, Observation, p. 177]. Thus, if $H=H_{0} \times H_{1}, H_{0}, H_{1}$ are any two nontrivial groups with $H_{0}$ is a finite group with trivial centre and if $H_{1}$ is torsionless, then $H$ is freely indecomposable and admits finite index characteristic subgroup, namely $H_{1}$. To see this we note that (i) any automorphism of $H$ maps $H_{0}$ to itself since $H_{1}$ is torsion-free, the centralizer of $H_{1}$ in $H$ contains $H_{0}$, and, (iii) the only element of $H_{0}$ in the centralizer of any element $\left(h_{0}, h_{1}\right) \in H$ is the trivial element. So $H_{1}$ is characteristic in $H$. Therefore we see that the hypotheses on the free factors of $G$ in Theorem 1 hold for a large family of groups.

Theorem 5 follows easily from Theorem 1 using the fact that the fundamental group of a compact three-manifold is residually finite. (See [T, Theorem 3.3], [H2]).

We should point out that Fel'shtyn outlined in $[\mathrm{F}]$ the main steps of a proof which shows that finitely generated non-elementary relatively hyperbolic groups have property $R_{\infty}$. This proof relies on group actions on $\mathbb{R}$-trees and other notions from geometric

\footnotetext{
${ }^{1}$ We work in the PL or smooth category. Note that every three-manifold admits (unique) PL and smooth structures.
} 
group theory. Thus Fel'shtyn's result will imply that any finite free product of freely indecomposable finitely generated groups has property $R_{\infty}$ from which Theorem 5 will follow. On the other hand, Theorem 1 does not assume that the free factors are finitely generated and the proof uses elementary techniques from combinatorial group theory. Hence, Theorem 1 does not follow from the result of $[\mathrm{F}]$. For instance, if $G$ is a freely indecomposable torsion-free group containing a proper finite index characteristic subgroup and if $H=\bigoplus_{p} \mathbb{Z}_{p}$ where $p$ varies over the set of all primes, then $G * H$ has property $R_{\infty}$ while $H$ is not finitely generated (see also $[\mathrm{SW}]$ ).

\section{ThE $R_{\infty}$-PROPERTY OF A FREE PRODUCT}

Our goal here is to establish the $R_{\infty}$-property for a free product $G=G_{1} * \cdots * G_{n}, n \geq 2$, for a wide class of groups $G_{i}$. The main tool will be the Kuroš theorem that reveals the structure of a subgroup of a free product. The strategy of proof would be to first establish our goal when all the $G_{i}$ are finite. Here the case $n=2$ is well-known. We then reduce the general case, under suitable hypotheses on the $G_{i}$, to the case of free product of finite groups.

We begin by recalling the Kuroš subgroup theorem. Let $G$ be a free product of groups $G=G_{1} * \cdots * G_{n}$ and let $K$ be a subgroup of $G$. Then $K$ is itself a free product of groups

$$
K=F_{0} * H_{1} * \cdots * H_{n}
$$

where each $H_{j}$ is a free product of a family of subgroups $\left\{\alpha_{i, j} H_{i, j} \alpha_{i, j}^{-1}\right\}_{i \in J_{j}}$ of $G$ for suitable elements $\alpha_{i, j} \in G$ and suitable subgroups $H_{i, j} \leq G_{j}, i \in J_{j}$ for some indexing set $J_{j}, 1 \leq$ $j \leq n$.

The following lemma is a standard application of the Kuroš subgroup theorem. We include a proof for the sake of completeness.

Lemma 2. Let $G=G_{1} * \cdots * G_{n}$ where each $G_{i}, 1 \leq i \leq n$ is a finite non-trivial group. Then $G$ is virtually free and hence has the $R_{\infty}$-property if $n \geq 2$.

Proof. The statement that $G$ is virtually free is trivially valid when $n=1$. So assume that $n \geq 2$. We consider the kernel of projection $\eta: G \rightarrow G_{1} \times \cdots \times G_{n}$, denoted $K$. Note that $\eta$ maps any conjugate of $G_{i}$ isomorphically onto $G_{i}$. Therefore, if $H_{i}$ is a subgroup of $G_{i}$ and $g \in G$, then $\eta\left(g H_{i} g^{-1}\right)$ maps onto $H_{i}$. It follows that, writing $K=F_{0} * K_{1} * \cdots * K_{n}$ as in $(*)$, we see that $K_{i}$ are trivial for all $i$. Therefore $K=F_{0}$ is a free group. Since $G / K=\prod G_{i}$ is finite, the index of $K$ in $G$ is finite. Since $G$ is finitely generated, the same is true of $K$.

If $n=2$ and $G_{1} \cong G_{2} \cong \mathbb{Z}_{2}$, then $G$ is infinite dihedral and it is known that $G$ has the $R_{\infty}$-property (see [GW]). In all other cases, with $n \geq 2, K$ is a non-abelian free group of finite rank. It follows that $G$ is finitely generated non-elementary word hyperbolic and thus has the $R_{\infty}$-property by [LL]. 
We say that a nontrivial group is freely indecomposable if it cannot be expressed as a free product of two nontrivial groups. The only nontrivial free group which is freely indecomposable is the infinite cyclic group.

If $\alpha: G \rightarrow H$ is an isomorphism and if $C \subset G$ is a characteristic subgroup of $G$, then $\alpha(C)$ is characteristic subgroup of $H$ which is independent of the choice of $\alpha$. Indeed, if $\beta: G \rightarrow H$ is another isomorphism then $\beta \circ \alpha^{-1}: H \rightarrow H$ is an automorphism. Since $\alpha(C)$ is characteristic, we have $\alpha(C)=\beta \circ \alpha^{-1}(\alpha(C))=\beta(C)$.

Lemma 3. Let $G=G_{1} * \cdots * G_{n}$ where each $G_{j}, 1 \leq j \leq n$, is freely indecomposable and not infinite cyclic. Let $C_{j} \subset G_{j}$ be a characteristic subgroup of $G_{j}, 1 \leq j \leq n$. Fix an isomorphism $\alpha_{i j}: G_{i} \rightarrow G_{j}$ whenever $G_{i}, G_{j}$ are isomorphic. Then the subgroup $K$ of $G$ generated by the family $\mathcal{C}$ of subgroups $g C_{j} g^{-1}, g \alpha_{i j}\left(C_{i}\right) g^{-1} \subset G, g \in G, 1 \leq i, j \leq n$, is characteristic in $G$.

Proof. Evidently $K$ is normal in $G$ since the family $\mathcal{C}$ is closed under conjugation. We need only show that the $\mathcal{C}$ is closed under any automorphism of $G$.

Let $\phi: G \rightarrow G$. Consider the subgroup $\phi\left(G_{j}\right)$. Since $G_{j}$ is freely indecomposable and is not infinite cyclic, the same is true of $\phi\left(G_{j}\right)$. By the Kuroš subgroup theorem, $\phi\left(G_{j}\right)$ is contained in $g_{j} G_{k_{j}} g_{j}^{-1}$ for some $k_{j} \leq n$ and $g_{j} \in G$. Therefore $\phi(G)=\phi\left(G_{1}\right) * \cdots *$ $\phi\left(G_{n}\right) \subset g_{1} G_{k_{1}} g_{1}^{-1} * \cdots * g_{n} G_{k_{n}} g_{n}^{-1} \subset G$. Since $\phi(G)=G$ we must have the equality $\phi\left(G_{j}\right)=g_{j} G_{k_{j}} g_{j}^{-1}$ for all $j$. In particular $\left.\left.\iota_{g_{j}^{-1}}\right|_{G_{k_{j}}} \circ \phi\right|_{G_{j}}: G_{j} \rightarrow G_{k_{j}}$ is an isomorphism, which we shall denote by $\kappa_{j}$. Here $\iota_{g}$ denotes the inner automorphism $x \mapsto g x g^{-1}$ of $G$.

Let $A_{j} \subset G_{j}$ be any characteristic subgroup of $G_{j}$. Then $\kappa_{j}\left(A_{j}\right)=\alpha_{j k_{j}}\left(A_{j}\right)$. Therefore $\phi\left(A_{j}\right)=g_{j}\left(\alpha_{j k_{j}}\left(A_{j}\right)\right) g_{j}^{-1}$.

Taking $A_{j}$ to be $C_{j}$ or $\alpha_{i j}\left(C_{i}\right)$, it follows that the family $\mathcal{C}$ is closed under any automorphism of $G$. Hence $K$ is characteristic in $G$.

Remark 4. (i) In our application, we shall choose the characteristic subgroups $C_{j}$ so that whenever $G_{i}$ and $G_{j}$ are isomorphic, $C_{i}$ corresponds to $C_{j}$ under an isomorphism $G_{i} \rightarrow G_{j}$. In this case $K \subset G$ is generated as a normal subgroup by the finite collection of subgroups $C_{j}, 1 \leq j \leq n$.

(ii) We remark that a finite index subgroup of a freely indecomposable group is not necessarily freely indecomposable. For example $S L(2, \mathbb{Z})$ is virtually free with a finite index non-abelian free subgroup but is freely indecomposable.

Proof of Theorem 1: By relabelling if necessary, we assume that (i) $G_{1}, \ldots, G_{n}$ are the free factors of $G$ such that either $G_{i}$ is infinite cyclic or is isomorphic to one of the groups $G_{1}, G_{2}$, and, (ii) the groups $G_{j}$ is not isomorphic to any of the groups $G_{1}, G_{2}, \mathbb{Z}$, for $n<j \leq k$. Note that $n \geq 2$.

Let $K$ be the kernel of the natural projection $G \rightarrow G_{1} * \cdots * G_{n}$ that maps each $G_{i}$ identically onto $G_{i}, 1 \leq i \leq n$ and maps $G_{j}$ to the trivial group for $j>n$. Then, by 
Lemma $3, K$ is characteristic. To show that $G$ has the $R_{\infty}$-property, we need only prove that $G_{0}:=G_{1} * \cdots * G_{n}$ has the $R_{\infty}$ property.

The proof will be divided into three cases depending on the number of groups $G_{i}, 1 \leq$ $i \leq n$, that are isomorphic to $\mathbb{Z}$ being zero, or one or at least two. We shall denote this number by $r$. Relabelling if necessary, we assume that $G_{i} \cong \mathbb{Z}$ if $1 \leq j \leq r$ in case $r>0$.

Let $C_{i} \subset G_{i}$ be a proper finite index characteristic subgroup of $G_{i}$. We assume, as we may, that whenever $G_{i} \cong G_{j}$, then $C_{j}$ corresponds to $C_{i}$ (under an isomorphism $G_{i} \rightarrow G_{j}$ ).

Case 1: Suppose that none of the $G_{i}$ is infinite cyclic. Set $\bar{G}_{i}=G_{i} / C_{i}, 1 \leq i \leq n$, and let $\bar{G}=\bar{G}_{1} * \cdots * \bar{G}_{n}$. Let $K_{0}$ be the kernel of the natural projection $G_{0} \rightarrow \bar{G}_{0}$. Then $K_{0}$ is normally generated by the finite collection of subgroups $\left\{C_{j} \mid 1 \leq j \leq n\right\}$. Hence by Lemma $3, K_{0}$ is characteristic in $G_{0}$. By Lemma $2, \bar{G}_{0}$ has the $R_{\infty}$-property. It follows that $G_{0}$ has the $R_{\infty}$-property.

Case 2: Suppose that $r>1$ so that $G_{i} \cong \mathbb{Z}$ for $1 \leq j \leq r$ and $G_{j} ¥ \mathbb{Z}$ for $j>n$. If $r=n$, in view of the fact that $n \geq 2, G_{0}$ is a non-abelian free group of finite rank and so has the $R_{\infty}$ property.

So suppose that $2 \leq r<n$. Set $A:=G_{1} * \cdots * G_{r}, B:=G_{r+1} * \cdots * G_{n}$ so that $G_{0}=A * B$ where $A$ is a non-abelian free group of rank $r$. Let $K_{1}$ be the kernel of the projection $G \rightarrow$ $A$. Then $K_{1}$ is the free product of the family of groups $\mathcal{C}=\left\{g G_{j} g^{-1} \mid g \in G, r<j \leq n\right\}$. Since each $G_{j}, j>r$, is indecomposable and not infinite cyclic, under any automorphism of $G_{0}, G_{j}$ is mapped to a conjugate of a $G_{i}$ isomorphic to $G_{j}$ where $r<i \leq n$. It follows that $\mathcal{C}$ is stable by any automorphism of $G_{0}$. Therefore $K_{1}$ is characteristic in $G_{0}$. Since $A$ is a free non-abelian group of finite rank, it has the $R_{\infty}$-property. It follows that $G_{0}$ also has the $R_{\infty}$-property.

Case 3: Suppose that $r=1$, that is, $G_{1} \cong \mathbb{Z}, G_{j} \neq \mathbb{Z}$ for $2 \leq j \leq n$. We consider the canonical projection $\eta: G_{0} \rightarrow G_{1} * \bar{B}$ where $\bar{B}$ is the free product of $G_{i} / C_{i}, 2 \leq i \leq n$. The kernel $K_{3}:=\operatorname{ker}(\eta)$ is generated by the collection $\left\{g C_{j} g^{-1} \mid g \in G_{0}, j \geq 2\right\}$. By Lemma $3, K_{3}$ is characteristic in $G_{0}$ in view of our hypothesis on the $C_{j}$. (See Remark 4 (i).) Now $\bar{B}$ is nontrivial and virtually free by Lemma 2, possibly finite. It follows that $G_{1} * \bar{B} \cong \mathbb{Z} * \bar{B}$ has a non-abelian free group of finite rank as a finite index subgroup. So $G_{1} * \bar{B}$ has the $R_{\infty}$-property. Since $K_{3}$ is characteristic in $G_{0}$, it follows that $G_{0}$ also has the $R_{\infty}$-property.

Thus in all cases, $G_{0}$ has the $R_{\infty}$-property as was to be shown.

As an immediate corollary, we obtain

Theorem 5. Let $M$ be a non-prime compact connected three-manifold. Then $\pi_{1}(M)$ has the $R_{\infty}$-property.

Proof. Since $M$ is not prime, it admits a prime decomposition: $M=M_{1} \# \cdots \# M_{k}$ where each $M_{i}$ is a prime manifold and $k \geq 2$. Thus $\pi_{1}(M)=\pi_{1}\left(M_{1}\right) * \cdots * \pi_{1}\left(M_{k}\right)$. (See [H1, Chapter 3].) Note that since $M_{i}$ is prime, $\pi_{1}\left(M_{i}\right)$ is freely indecomposable in view of [H1, Theorem 7.1]. Also, it is known that $\pi_{1}\left(M_{i}\right)$ is residually finite as a consequence 
of the geometrization theorem and the work of Thurston [T, Theorem 3.3]. (See also [H2].) So we may take $G_{i}$ to be $\pi_{1}\left(M_{i}\right)$ in Theorem 1 and we see that $\pi_{1}(M)$ has the $R_{\infty}$-property.

Remark 6. (i) The same arguments as above also yield the following: if $M$ is a connected sum $M_{1} \# \cdots \# M_{r}, r \geq 2$, where each $M_{j}$ is a connected $n$-manifold $(n \geq 3)$ and $M_{1}$ and $M_{2}$ each admits a non-trivial finite characteristic cover, then $\pi_{1}(M)$ has the $R_{\infty}$ property. (A cover is characteristic if it corresponds to a characteristic subgroup of the fundamental group.)

(ii) Let $P \subset \mathbb{N}$ be a nonempty proper subset of primes. Suppose that $p \notin P$. Let $\mathbb{Z}(P) \subset \mathbb{Q}$ be subring $\mathbb{Z}[1 / q \mid q \in P]$. Since $p \notin P$, we have a natural surjective ring homomorphism $\mathbb{Z}(P) \rightarrow \mathbb{Z} / p \mathbb{Z}$. The kernel is a proper characteristic subgroup of $\mathbb{Z}(P)$. (It consists precisely of elements which are expressible as $p x, x \in \mathbb{Z}(P)$.) Evidently $\mathbb{Z}(P)$ is freely indecomposable. It is readily seen that $\mathbb{Z}(P)$ is not isomorphic to $\mathbb{Z}\left(P^{\prime}\right)$ if $P \neq P^{\prime}$ so the collection of such groups has cardinality the continuum. It is known that $\mathbb{Z}(P)$ is the fundamental group of an open three-manifold $M(P)$, which is in fact an aspherical space. This can be derived from constructing a non-compact 3-manifold (in fact, the complement of a solenoid in $\mathbb{S}^{3}$ ) whose fundamental group is $\mathbb{Q}$ (see e.g. [EM, p.209]). If $M_{j}:=M\left(P_{j}\right), 1 \leq j \leq k$, are such manifolds (where we do not assume that the $P_{j}$ are pairwise distinct) then their connected sum $M_{1} \# \cdots \# M_{k}$ is an open three-manifold whose fundamental group has the $R_{\infty}$-property, provided $k \geq 2$ and at least two sets, say, $P_{1}, P_{2}$ are nonempty proper subsets of the set of all primes.

Remark 7. (i) Let $M$ be a closed connected three-manifold such that $\pi_{1}(M)$ is infinite cyclic. Then $M$ is prime. We claim that $M$ is a 2-sphere bundle over the circle. If $M$ is irreducible, then by the sphere theorem, $\pi_{2}(M)$ is trivial. Since $\pi_{1}(M)$ is infinite, it is a $K(\mathbb{Z}, 1)$-space. Therefore it is homotopic to a circle. This is a contradiction since $H_{3}\left(M ; \mathbb{Z}_{2}\right) \cong \mathbb{Z}_{2}$. So $M$ is not irreducible. By [H1, Lemma 3.13] $M$ is a 2 -sphere bundle over the circle.

(ii) When a three-manifold $M$ admits a geometric structure, in some cases it is known whether or not $\pi_{1}(M)$ has the $R_{\infty}$-property. For example, when $M$ admits spherical geometry, the fundamental group is finite and it is trivial that $\pi_{1}(M)$ does not have the $R_{\infty}$-property. On the other hand, when the manifold admits hyperbolic geometry, then $\pi_{1}(M)$ has the $R_{\infty}$-property as an immediate consequence of the work of Levitt and Lustig [LL]. In the case of $\mathbb{S}^{2} \times \mathbb{R}$-geometry, we see that $\pi_{1}\left(\mathbb{S}^{2} \times \mathbb{S}^{1}\right) \cong \mathbb{Z}$ does not have $R_{\infty^{-}}$ property whereas $\pi_{1}\left(\mathbb{R} P^{3} \# \mathbb{R} P^{3}\right) \cong \mathbb{Z}_{2} * \mathbb{Z}_{2}$ has the $R_{\infty}$-property; see [GW]. It can be shown that the fundamental groups of Seifert fibre spaces have the $R_{\infty}$-property provided the base surface has genus at least 2. However the complete classification for geometric three-manifolds will take us too far a field. It will be carried out in a forthcoming article [GSW].

In general, by Thurston's geometrization conjecture (Perelman's theorem), for any orientable prime three-manifold $M$ that is not geometric, the fundamental group of $M$ is an 
iterated amalgamated free product of fundamental groups of geometric manifolds where the amalgamating group is $\mathbb{Z}^{2}$. Our approach to Theorem 1 fails in this setting.

Acknowledgement: We thank Mihalis Sykiotis for pointing out some gaps in our arguments in an earlier version of this paper.

\section{REFERENCES}

[EM] B. Evans and L. Moser, Solvable fundamental groups of compact 3-manifolds. Trans. Amer. Math. Soc. 168 (1972), 189-210.

[F] A. Fel'shtyn, New directions in Nielsen-Reidemeister theory Topology Appl. 157 (2010), no. 10-11, $1724-1735$.

[GW] D. Gonçalves and P. Wong, Twisted conjugacy classes in nilpotent groups. J. Reine Angew. Math. 633 (2009), 11-27.

[GSW] D. Gonçalves, P. Sankaran, and P. Wong, Twisted conjugacy for geometric 3-manifolds, in preparation.

[H1] J. Hempel, 3-manifolds. Reprint of the 1976 original. AMS Chelsea Publishing, Providence, RI, 2004.

[H2] J. Hempel, Residual finiteness for 3-manifolds. Combinatorial group theory and topology (Alta, Utah, 1984), 379-396, Ann. of Math. Stud., 111, Princeton Univ. Press, Princeton, NJ, 1987.

[LL] G. Levitt and M. Lustig, Most automorphisms of a hyperbolic group have very simple dynamics. Ann. Sci. École Norm. Sup. (4) 33 (2000), no. 4, 507-517.

[LS] R. Lyndon and P. Schupp, Combinatorial group theory. Springer-Verlag, Berlin, 1977.

[MS] T. Mubeena and P. Sankaran, Twisted conjugacy classes in abelian extensions of certain linear groups. Canad. Math. Bull. 57 (2014), no. 1, 132-140.

[SW] P. Sankaran and P. Wong, Twisted conjugacy and commensurability invariance, https://arXiv:2001.02027v1 [math.GR], 7 Jan 2020.

[T] W. P. Thurston, Three-dimensional manifolds, Kleinian groups and hyperbolic geometry. Bull. Amer. Math. Soc. (N.S.) 6 (1982), no. 3, 357-381.

Instituto de Matemámatica e Estatística da Universidade de São Paulo, Departamento de Matemática, Rua do Matão, 1010 CEP 05508-090, São Paulo-SP, Brasil

E-mail address: dlgoncal@ime.usp.br

Chennai Mathematical Institute, H1 SipCOT it Park, Siruseri, Kelambakkam, 603103, INDIA

E-mail address: sankaran@cmi.ac.in

Department of Mathematics, Bates College, Lewiston, Maine, UsA

E-mail address: pwong@bates.edu 